\title{
Uterine Didelphys Associated With Obstructed Hemivagina and Ipsilateral Renal Anomaly (OHVIRA) Syndrome: a Case Report
}

\author{
Parvin A ${ }^{1}$, Khan B S², Prof. Brig Gen (Retd) Alam J33, Ruby F A4, Iqbal T J5
}

\begin{abstract}
A 30 year old nulligravida female reported to the fertility centre of AHD with the complaints of primary infertility for three and half years and spasmodic dysmenorrhoea. There is also history of progressively increasing right lower abdominal pain as well as discomfort which was cyclically associated with the onset of menses. Transabdominal sonography showed-'Endometrial splitting into two at the fundus-suggesting bicornuate uterus. Echogenic soft tissue in the cervical canal due to blood clots. Non visualized right kidney. Mildly enlarged left kidney'. HSG done outside AHD suggestive of unicornuate uterus with single fallopian tube. IVU showed non visualized right kidney. Normally excreting left kidney. TVS showed normal sized septated nulliparus uterus with homogeneous myometrium and thick endometrium with proliferative phase echo. Mildly enlarged right ovary with mildly distended right tube. Mild collection adjacent to the vagina. Then the patient came to the gynaecology dept of AHD from where she was sent to our Radiology department to undergo MRI of pelvis. The MRI showed uterine didelphys. Obstructed hemivagina (right) with hematocolpos extended upto pelvic brim along right and posterior aspect of uterus through anomalous dilated remnant of right lower ureter with ipsilateral renal agenesis. Patient was diagnosed as OHVIRA syndrome radiologically.
\end{abstract}

\section{Introduction}

A didelphic uterus with an obstructed hemivagina and ipsilateral renal agenesis is a rare congenital anomaly constituting $0.16-10 \%$ of all Mullerian duct abnormalities(MDA). ${ }^{2}$ This syndrome of obstructed hemivagina and ipsilateral renal anomaly was initially reported in 1922 and is known as Herlyn-Werner-Wunderlich syndrome or more recently, by the acronym OHVIRA. ${ }^{3-6}$ Among the seven types of Mullerian ducts anomaly our case matched with class iii Mullerian ducts anomaly radiologically.

\section{Case report}

A 30 year old nulligravida female reported to the fertility centre of AHD with the complaints of primary infertility for three and half years and spasmodic dysmenorrhoea. She had attained menarche at the age of 13 years, had regular cycles of 2 days with flow for 3-5 days (scanty). There is also history of pprogressively increasing right lower abdominal pain as well as discomfort which was cyclically associated with the onset of menses. On clinical evaluation it was found that patient was moderately built and had normal vital signs.The blood profile was unremarkable with normal renal function. Transabdominal sonography among available previous documents showed 'Endometrial splitting into two at the fundus suggesting bicounuate uterus. Echogenic soft tissue in the cervical canal due to blood clots. Non visualized right kidney. Mildly enlarged left kidney'. HSG was suggestive of unicornuate uterus with single fallopian tube. IVU showed non visualized right kidney. Normally excreting left kidney. TVS showedNormal sized septate nulliparus uterus with homogeneous myometrium and thick endometrium with proliferative phase echo. Mildly enlarged right ovary with mildly distended right tube. Then the patient was sent to our Radiology department to undergo MRI of pelvis during the time of menstruation for further evaluation. The MRI was performed by 1.5 Tesla 
Uterine Didelphys Associated With Obstructed Hemivagina and Ipsilateral

Renal Anomaly (OHVIRA) Syndrome

MR clinical scanner which showed features consistent with uterine didelphys. Obstructed hemivagina (right) with hematocolpos extended upto pelvic brim along right and posterior aspect of uterus through anomalous dilated remnant of right lower ureter with ipsilateral renal agenesis. Patient was diagnosed as OHVIRA syndrome radiologically.

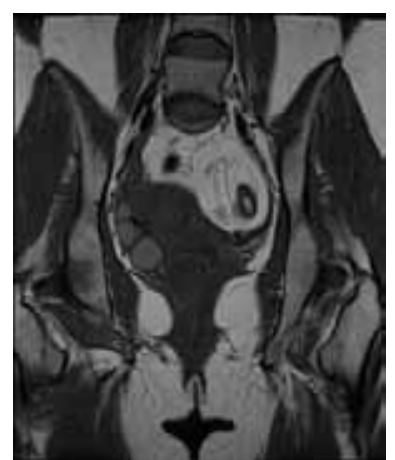

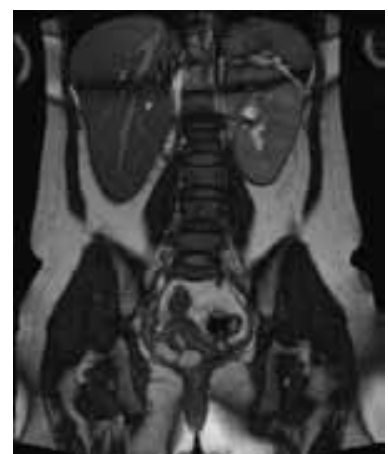

b

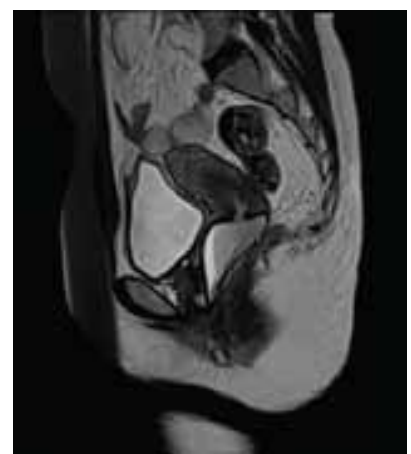

C

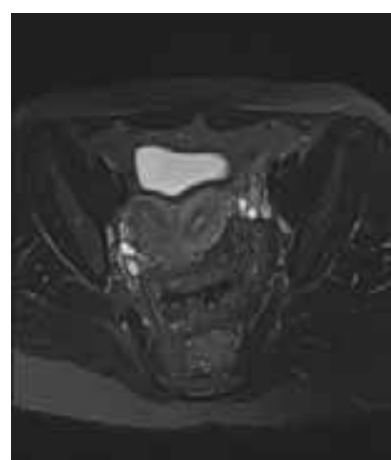

d

Fig : (a) Dilated lower right ureter(coronal T1WI), (b) Ipsilateral renal agenasis (coronal T2WI), (c) Obstructed hemivagina with hematocolpos (sagital T2WI) and (d) Uterine didelphys (axial T2WI).

\section{Discussion}

Anomalies of the internal female reproductive tract referred to as the Mullerian duct anomalies, account for about $7 \%$ of female population. ${ }^{1} \mathrm{~A}$ didelphic uterus with an obstructed hemivagina and ipsilateral renal agenesis is a rare congenital anomaly constituting $0.16-10 \%$ of all Mullerian duct abnormalities. ${ }^{2}$ This syndrome of obstructed hemivagina and ipsilateral renal anomaly was initially reported in 1922 and is known as Herlyn-Werner-Wunderlich syndrome or more recently, by the acronym OHVIRA. 3-6

Mullerian duct anomalies are congenital anatomic abnormalities that arise from non development or non fusion or failed resorption of Mullerian ducts. They are particularly important because they are associated with an increased risk of infertility, menstrual disorders and obstetric complications. The first stage of mullerian duct development begins at an approximately 6 weeks gestational age when the paired mullerian ducts invaginate and then grow caudally and cross over the Wolffian ducts to meet at the midline. The subsequent three phases (fusion, resorption and vaginal induction) proceed in an orderly fashion from 9th to the 22nd gestational week. Mullerian duct anomalies are classified according to the American fertility society ${ }^{7,8}$ criteria as follows:

Class i or uterine hypoplasia or agenesis:

Class ii or unicornuate uterus: A banana shaped uterus with a single fallopian tube. A rudimentary horn (communicating or non communicating) may be present.

Class iii or uterine didelphys: Two complete uteruses, each with its own cervix. A sagittal vaginal septum is seen in the majority of cases. Class iv or bicornuate uterus: Two uterine cavities with one cervix with an intercornual distance of $>4 \mathrm{~cm}$ and convexity fundal contour or an external fundal cleft of $>1 \mathrm{~cm}$ in depth. ${ }^{7}$

Class $\mathbf{v}$ or septate uterus: A fibrous septum is seen that appear hypointense on T2W images. While the muscular septum appears intermediate 
in intensity. MRI criteria includes a convex or flat external fundal contour or external fundal cleft of $<1 \mathrm{~cm}$ in depth. ${ }^{9}$

Class vi or arcuate uterus: It is a normal variant and is characterized by convex contour of fundus with fundal endometrial indentation.

Class vii or diethylbestrol induced: exposure to this synthetic estrogen antenatally can result in a T shaped, hypoplastic and constricted uterus.

Our case matched with class iii Mullerian ducts anomaly.

A didelphic uterus is suggestive of an embryologic arrest occuring during the 8th week of gestation which ultimately affects the Mullerian and Metanephric ducts. ${ }^{10}$ However, if one of the Wolffian ducts is absent, the kidney and ureter on the ipsilateral side will fail to fuse at midline.This process may occur completely or incompletely. If the failure to fuse is complete, then a uterine didelphys is formed. The mullerian duct on the side lacking the Wolffian duct, displaces itself laterally and cannot come into contact with the urogenital sinus in the centre resulting in a blind sac, imperforate or obstructed vagina. However the distal part of the vagina, originating from the urogenital sinus is not affected. ${ }^{10}$ Each ureteric bud develops from the Wolffian duct which is ultimately responsible for the development of the kidney. The ureteric bud grows dorsocranially into the metanephric blastema inducing differentiation of the metanephric nephrons. If the uretetric bud fails to either from or make contact with the metanephric blastema, the kidney on that side will fail to develop. ${ }^{10}$

Very rarely ectopic ureter can be associated with this syndrome either inserting into the obstructed vaginal cavity or a Gartner's duct cyst on the side of congenitally absent ipsilateral kidney. ${ }^{11}$ Renal tract anomalies associated with
MDA in upto $30 \%$ of cases due to the close embryologic relationship between the paramesonephric and mesonephric ducts. The most common renal tract anomaly associated with MDA is renal agenesis with right sided prevalence ${ }^{15,16,17}$ which was also prevalent in our case.

As in most of the cases, our patient also presented with cyclical pain during menstruation and then progressing into mass per abdomen. Complications like endometriosis, adhesions and PID due to hematocolpos can be prevented if an early diagnosis can be made. Sonography is frequently the first imaging modality in assessing genitourinary pathologies. In our case, the ectopic ureter was misinterprited as hydrosalpinx in TVS.

Preoperative MRI diagnosis of ectopic ureter associated with OHVIRA is unique. MRI is an excellent modality for evaluating the frequently complex genitourinary anomalies, given its multiplanner capability, superior tissue characterization and the lack of ionizing radiation. Delays in diagnosis have been attributed to lack of understanding of this condition by radiologists, gynecologists and pediatricians. Delayed diagnosis can lead to complications that include endometriosis, adhesions, infertility and infectious complications arising from chronic cryptomenorrhoea.

\section{Conclusion}

The diagnosis of OHVIRA should be suspected in cases of women presenting with pelvic pain and pelvic mass with ipsilateral renal agenesis. Systematic analysis of the literature suggests management of these cases consists mainly of vaginoplasty with excision of vaginal septum in order to prevent the long term complications of 


\section{Uterine Didelphys Associated With Obstructed Hemivagina and Ipsilateral Renal Anomaly (OHVIRA) Syndrome}

recurrent pyocolpos and infertility from chronic cryptomenorrhoea. Those cases where an ectopic ureter inserts into the obstructed hemivagina, laparoscopic surgical ligation vs surgical removal is advocated to prevent recurrent fluid accumulation in the obstructed hemivagina.

\section{References}

1. Vallerie Amy M, Breech Lesley L. Update in mullerian anomalies: diagnosis, management and outcomes. Curr opin obstet gyneco. 2010;22(5):381-387.

2. Adair ii l, Georgiades M Osborne R. Uterus didelphys with unilateral distal vaginal agenesis and ipsilateral renal agenesis: common presentation of an unusual variation. Journal of Radiology. Case reports. 2011;5(1):1-8.

3. Smith NA. Obstructed hemivagina and ipsilateral renal anomaly syndrome. 2007 Apr;87(4):918-22.

4. Gholoum S. Management and outcome of patients with combined vaginal septum, bifid uterus and ipsilateral renal agenesis. J Pediatr Surg. 2006 May;41(5):987-92.

5. Herlyn U,Werner H. Simultaneous occurence of an open Gartner cyst, a homolateral aplasia of the kidney and a double uterus as a typical syndrome of abnormalities. Geburtshilfe Frauenheilkd. 1971 Apr;31(4):340-7.(Pub Med).

6. Underlich. Unusual form of genital malformation with aplasia of right kidney. Zentralbl Gynakol. 1976;98(9):559-62.(Pub Med).

7. Buttram VC, Gibbons WE. Mullerian anomalies: a proposed classification (an analysis of 14 cases). Fertil Steril. 1979;32:40-46.

8. The American Fertility Society. Classifications of adnexal adhesions, distal tubal obstruction, tubal occlusion secondary to tubal ligation, tubal pregnancies, mullerian anomalies and intrauterine adhesions.
9. Pellerito JS, McCarthy SM, Doyle MB, Glickman MG, DeCherney AH. Diagnosis of uterine anomalies: Relative accuracy of MRI, endovaginal sonography and hysterosalpingography. Radiology. 1992;183:795-800.

10. Vercellini P, Daguati R, Somigliana E et al. Asymmetric lateral distribution of obstructed hemivagina and renal agenesis in women with uterus didelphys:institutional case series and a systematic literature review. Fertility sterility. 2007;7():719-2. PMID 1730731.

11. Boram H,Herndon C, Rosen $M$ et al. Uterine didelphys associated with obstructed hemivagina and ipsilateral renal anomaly (OHVIRA) syndrome: Radiology case reports. 2010.Issue 1.

12. Duong DT Shortlife LM.. A case of ectopic dysplastic kidney and ectopic ureter diagnosedby MRI. Nat Clin Practrrol. 2008;5:632-636.

13. Shibata T, Nonomura Kkakizaki H. A case of unique communication between blind-ending ectopic ureter and ipsilateral hemi-hematocolpometra in uterine didelphys. J Urol.1995;153:1208-1210.

14. Sheih CP, Chiang CD, Liao YJ . Ultrasonic detection of a single vaginal ectopic ureter inserted into an imperforate hemivagina. J Clin ultrasound. 1997;25:133-135.

15. Gholoum S, Puligandla PS, Hui T, Su W, Quiros E. Management and outcome of patients with combined vaginal septum, bifid uterus and ipsilateral renal agenesis. JPediatr Surg. 2006;1(5):98-92.

16. Burgis J: Obstructive Mullerian anomalies. case report, diagnosis and management. Am J Obstet Gynaecol. 2001;185:338-44.

17. Gruenwald P. Relation of growing Mullerian duct to the Wolffian duct and its importance for the genesis of malformation. Anat Rec. 191;81:1-20. 